\title{
A Deontic Logic Framework Allowing for Factual Detachment*
}

\author{
Christian Straßer \\ Centre for Logic and Philosophy of Science, Ghent University (UGent) \\ christian.strasser@UGent.be \\ June 22, 2009
}

\begin{abstract}
Due to the fact that a large number of our ethical and behavioural norms have a conditional form, it is of great importance that deontic logics give an account of deontic commitments of the form " $A$ commits you to do/bring about $B$ ". It is commonly agreed that monadic approaches are only suboptimal for this task since they have several shortcomings, for instance their falling short of giving a satisfactory account of "Strengthening of the antecedent" or their difficulties in dealing with contrary-to-duty paradoxes. While dyadic logics are more promising in these respects, they have been criticized for not being able to model "detachment" ([8]): $A$ and the commitment under $A$ to do $B$ implies the actual obligation to do $B$. "We seem to feel that detachment should be possible after all. But we cannot have things both ways, can we? This is the dilemma on commitment and detachment." ([15], p. 658)

In this paper I answer Åqvist's question with "Yes, we can" by proposing a general method to turn dyadic deontic logics in adaptive logics allowing for factual detachment. It is shown that two types of obligations may be detached, proper and instrumental, which in case of violated obligations differ. Furthermore, a concrete realization based on Lou Goble's conflict tolerant CDPM logics ([5]) is presented.
\end{abstract}

\section{Introduction}

While monadic deontic logics, i.e. logics employing unary obligation and permission operators, are powerful tools to model actual and general obligations, there are several shortcomings concerning their abilities to model conditional obligations. Since most of our moral or behavioural norms are in a conditional form it is very important to develop deontic logics which are able to tackle such complications. There are two canonical ways to represent conditional obligations of the type "Under condition $A$ you're obliged/committed to bring about $B$ " in unary logics: (i) by $A \supset \mathrm{O} B$ and (ii) by $\mathrm{O}(A \supset B)$. A lot of criticism of such ways of modelling commitment by use of a monadic operator has been published. Prominent arguments concern the appropriate representation of strengthening of the antecedent (SA),if $A$ commits you to do $B$ then $A$ and $C$ commit you to do $B$. In most situations the logic is obviously expected to validate (SA). But consider the following case (A):

\footnotetext{
${ }^{*}$ Research for this paper was supported by the Research Fund of Ghent University by means of Research Project 01G01907. I am indebted to Joke Meheus and Dunja Šešelja for comments on a former draft of this paper.
} 
A1 In general we're supposed not to eat with fingers.

A2 Eating asparagus we're allowed to eat with fingers.

If A1 is modelled by $T \supset O \neg f$, then $a \supset O \neg f$ is derivable. This obviously is in conflict with A2, $a \supset P f$, which is equivalent to $a \supset \neg \mathrm{O} \neg f$ since $P A$ is commonly defined as $\neg \mathrm{O} \neg A$. Similarly problematic is to use $\mathrm{O}(A \supset B)$ to represent the obligation $B$ under condition $A$. In the example we can derive by the inheritance principle $\mathrm{O}(a \supset \neg f)$ from $\mathrm{O}(\top \supset \neg f)$.

However, an argument concerning contrary to duty (CTD) obligations is commonly considered as the deathblow for any kind of effort in modelling conditional obligation in a monadic way. A prominent example is given by the so-called Chisholm Paradox (C) (cp. [2]):

C1 John ought to not impregnate Diane.

C2 If John impregnates Diane, he ought to marry her.

C3 If John doesn't impregnate Diane, he ought to not marry her.

C4 John impregnates Diane.

It has been argued that deontic paradoxes are due to the lack of a modelling of the temporal aspect of obligations (cp. [11]). However, Prakken and Sergot have in [13] presented a version of Chisholm's paradox which is entirely independent of any temporal parameters. This shows that earlier hopes to resolve all CTD puzzles by temporal deontic logics have to be abandoned. Furthermore it motivates research in non-temporal deontic logics which are able to tackle CTD puzzles.

It is commonly accepted that dyadic approaches can lead to satisfying solutions with respect to the CTD problems and, as for instance in the case of Goble's CDPM [5], there are also ways to tackle problems such as the ones related to strengthening of the antecedent. Nevertheless, dyadic approaches are criticized for not giving a satisfactory account of detachment. Detachment is, generally speaking, to derive the actual obligation to bring about $A$ from the commitment to bring about $A$ under condition $B$ and the fact $B .1$ Åqvist pessimistically stated "We seem to feel that detachment should be possible after all. But we cannot have things both ways, can we? This is the dilemma on commitment and detachment." ([15], p. 658) And indeed most of the existing dyadic logics do not allow for desired forms of detachment.

The logics presented in this paper are able to detach two types of obligations which are going to be analyzed in Section 2. In Section 3 I will present a generic method to turn dyadic deontic logics into adaptive logics that are able to model detachment. After having introduced the reader into Lou Goble's system CDPM in Section 4, I will present in Section 5 a concrete adaptive logic DCDPM based on this system constructed along the lines elaborated in Section 2 and Section 3. DCDPM is not only able to model detachment, but also to deal with deontic conflicts, i.e. situations in which we are committed to do $A$ as well as to do not$A$ or some $B$ which cannot be jointly realized with $A$. A prominent example is Sartre's pupil who had problems deciding if he should stay at home to support his ill mother or if he should rather fight the Nazis. Both obligations are mutually exclusive. Standard deontic logics lead in such cases to deontic explosion, namely to the derivability of all obligations. A logic that is able to deal with deontic dilemmas should, on the one hand, not have explosive characteristics in such cases and, on the other hand, still be able to derive as much as possible. Lou Goble presented a system CDPM based on a restriction of the inheritance principle that

\footnotetext{
${ }^{1}$ This is usually dubbed factual detachment. There is also deontic detachment (cp. [8]). I give a more formal account of both later.
} 
is able to satisfy both criteria. This is a good reason to demonstrate the adaptive handling of detachment on basis of it.

Finally, in Section 6 I suggest some enhancements of the logics.

\section{What to detach?}

This section clarifies our intuitions about what kind of obligations to detach from conditional obligations. There is a variety of conceptions concerning the types of obligations involved in certain setups and their relationships. Furthermore, scholars disagree about how and when to apply detachment.

Preliminarily it can be said that what should be detached are obligations that are in some sense 'actual' and that in some sense 'bind us'. These are obligations whose condition is fulfilled, and that are not 'cancelled' or 'destroyed' by other obligations. Especially the latter condition deserves a deeper analysis which I am going to provide in this section. The result will be that there are two types of obligations that we want to detach. I will dub them instrumental and proper obligations. In order to explicate these concepts I will first focus on two paradigmatical cases in which conditional obligations are in a sort of tension: specifity and contrary-toduty obligations. This will provide a good basis to entangle the discussion about the proper detachment in the literature.

Specifity Let us look at an example:

- Being served a meal, we are obliged not to eat with fingers.

- We're being served a meal.

We write $\mathrm{O}(\neg f \mid m)$ for the conditional obligation and $m$ for the second premise. We sometimes refer to $\neg f$ as being the conclusion and to $m$ as being the antecedent of the conditional obligation $\mathrm{O}(\neg f \mid m)$. Further we will read e.g. $\mathrm{P}(\neg f \mid m)$ as follows: 'Being served a meal, we're allowed not to eat with fingers.' It seems reasonable to apply factual detachment

$$
\frac{\mathrm{O}(A \mid B) B}{O A}
$$

to the two premises in order to derive the 'actual' obligation to not eat with fingers. However, we have to be careful. Norms and obligations are often stated in an elliptical way, i.e. the conditions are not precise but allow for exceptional contexts. Consider for instance our asparagus example (A1, A2), $\mathrm{O}(\neg f \mid \top)$ and $\mathrm{O}(f \mid a)$, or the weaker version, $\mathrm{O}(\neg f \mid \top)$ and $\mathrm{P}(f \mid a)$. Often scholars dub such settings specifity. Formally we can characterize specifity by $\mathrm{O}(A \mid B) \wedge \mathrm{P}(D \mid B \wedge C) \wedge \mathrm{P}(C \mid$ $B) \wedge B \wedge C$ where $D \vdash \neg A$. Note that in case $B \wedge C$ we have no intuitions to detach $\mathrm{O} A$ since $D$ is permitted in the more specific situation $B \wedge C$ by $\mathrm{P}(D \mid B \wedge C)$ and $D$ implies $\neg A$. Furthermore, in the context $B \wedge C$ our obligation $\mathrm{O}(A \mid B)$ is not violated, rather $\mathrm{P}(D \mid B \wedge C)$ describes an exception to it since we have $\mathrm{P}(C \mid B)$ which in a sense approves $B \wedge C$ and the permissions and obligations conditioned for it, such as $\mathrm{P}(D \mid B \wedge C)$. In Example $(\mathrm{A})$ a situation in which we are being served asparagus as well as the connected obligation $\mathrm{O}(f \mid a)$ are approved by $\mathrm{P}(a \mid \top)$. Thus $\mathrm{O}(f \mid a)$ should not be seen as a violation to $\mathrm{O}(\neg f \mid \top)$ but rather as an exception.

More formally we can define, a $B \wedge C$ is an exceptional context to $\mathrm{O}(A \mid B)$ iff there are $\mathrm{P}(C \mid B)$ and $\mathrm{P}(D \mid B \wedge C)$ where $D \vdash \neg A$. We say that $\mathrm{O}(A \mid B)$ is excepted in such a situation. In our example $a$ is an exceptional context to $\mathrm{O}(\neg f \mid \top)$, and $\mathrm{O}(\neg f \mid \top)$ is thus excepted.

We can contrast this with $B \wedge C$ being a violation context to $\mathrm{O}(A \mid B)$ iff (i) it is no exceptional context to $\mathrm{O}(A \mid B)$ and (ii) either (a) $A \vdash \neg C$ or (b) $(\mathrm{P}(D \mid B \wedge C)$ 
is valid and $A \vdash \neg D){ }^{2}$ In case of (a) $\mathrm{O}(A \mid B)$ is factually violated by $C$, in case of (b) it is violated by $\mathrm{P}\left(D \mid B \wedge C\right.$ ) (and not excepted!) ${ }^{3}$ In this sense violation contexts define sub-ideal situations, opposite of exceptional contexts, in which no obligation is violated. We say that $\mathrm{O}(A \mid B)$ is violated in $B \wedge C$ if $B \wedge C$ is a violation context to $\mathrm{O}(A \mid B)$. In order to give an example it is better to first introduce CTD-obligations.

Contrary-to-duty obligations While cases such as the asparagus example are usually considered as obligations overriding in certain contexts other obligations, something different seems to be going on in the following example $(\mathrm{G}): 4$

G1 Doe is in general obliged not to kill his mother. $-\mathrm{O}(\neg k \mid \mathrm{T})$

G2 However, if Doe kills his mother, he ought to kill her gently. $-\mathrm{O}(g \mid k)$

G3 Doe kills his mother.- $k$

Obligations of this kind are usually dubbed contrary-to-duty obligations. Formally: $\mathrm{O}(A \mid B \wedge C)$ is a (strong) CTD-obligation to $\mathrm{O}(D \mid B)$ iff $A \vdash \neg D$ and $A \vdash \neg C$. We call $\mathrm{O}(A \mid B)$ the primary and $\mathrm{O}(D \mid B \wedge C)$ the secondary obligation. Note that $A$ is inconsistent, both with the antecedent and the conclusion of $\mathrm{O}(D \mid B \wedge C)$. We define $\mathrm{O}(D \mid B \wedge C)$ as a weak CTD-obligation to $\mathrm{O}(A \mid B)$ iff only the latter is the case, i.e. $A \vdash \neg C$. An example (PA) is

PA1 You should not break your promise. $-\mathrm{O}(\neg b \mid \top)$

PA2 If you break your promise you are supposed to apologize. $-\mathrm{O}(a \mid b)$

Note that in both CTD-cases the primary obligation $\mathrm{O}(A \mid B)$ is (factually) violated if the antecedent of the secondary obligation is valid and $\mathrm{O}(A \mid B)$ is not excepted. For instance $k$ is a violation context to $\mathrm{O}(\neg k \mid T)$ and analogously for $b$ and $\mathrm{O}(\neg b \mid \top)$.

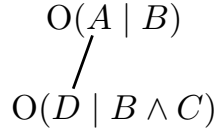

(i)

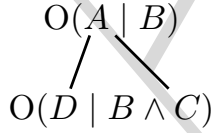

(ii)

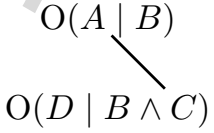

(iii)

Figure 1: (i) Specifity, (ii) strong CTD, (iii) weak CTD. The line indicates inconsistency between for instance $A$ and $D$ in (i).

The dissent in the literature There has been some discussion concerning factual detachment, especially with respect to (strong) CTD-cases such as the Forrester paradox (G). McNamara argues against detaching (strong) CTD-obligations:

So carte blance factual detachment seems to allow the mere fact that I will take an action in the future (killing my mother) that is horribly wrong and completely avoidable now to render obligatory another horrible (but slightly less horrible) action in the future (killing my mother gently). ([12], p. 268)

First note that for this paper I take it for granted that if an action to bring about $A$ is unavoidable, e.g. because of physico-causal reasons which are described by $X$,

\footnotetext{
${ }^{2}$ Torre in [19] defines $\neg A \wedge B$ to be a violation context for $\mathrm{O}(A \mid B)$. However, this distinguishes them too less from exceptional contexts: if we have $\neg f \wedge a$ then obviously we have $\neg f \wedge T$ which is a violation context in Torre's sense to $\mathrm{O}(\neg f \mid \top)$, whereas in our terminology this defines a exceptional context. Torre also points out that a violation context "distinguishes between ideal and varying sub-ideal contexts" (p. 3).

${ }^{3}$ The reader finds an example for (b) in our discussion of the Chisholm Paradox in Section 5.2.

${ }^{4}$ This version of the Forrester Paradox (cp. [4]) is taken from McNamara [12] (p. 243).
} 
then a conditional obligation $\mathrm{O}$ with conclusion $\neg A$ is excepted by $\mathrm{P}(A \mid X)$ and thus not violated. Vice versa this means, that if $A$ was avoidable but anyway takes place and $\mathrm{O}$ is not excepted for other reasons, $\mathrm{O}$ is violated. In the literature on CTD-obligations the primary obligation is usually considered to be violated and thus not to be excepted.

This, $\mathrm{O}(\neg k \mid T)$ being violated, is what McNamara has in mind in his criticism: in the case that Doe cannot be excused for killing his mother since it was 'completely avoidable', but he does it anyway, it seems absurd to detach an obligation to kill her gently since, after all, the primary obligation binds Doe despite him acting against it.

Wang in [22] however points out the importance of premise-dependency when evaluating detachments concerning obligations. He remarks: "the logic should construct the detachment inference in a way that makes the conclusion be evaluated in a premise-dependent way." In order to explicate this notion he reminds his readers of classical logic: here " $B$ is a logical consequence of $A$, iff if $A$ is true, then $B$ is true, where the evaluation of $B$ is given under the evaluation of $A$, i.e. $B$ is evaluated in the models where $A$ is true." In the Gentle Murderer case, the conclusion to kill the mother gently has the unintuitive appeal highlighted by McNamara mainly when interpreted in a premise-independent way, namely when we forget that the evaluation of the conclusion takes place in worlds/models in which Doe anyway kills her. Of course, isolated, i.e. independent from the evaluative contraint that he in fact kills her, the obligation to kill his mother gently is against our moral convictions. However, settling for a premise-dependent evaluation and accepting the premises G2 and G3, there is nothing which should cause any unease with our acceptance of the conclusion, that he should kill her gently ${ }_{5}^{5}$ Similarly, accepting the conclusion "Doe will win the lottery tomorrow." from premises (i) "If Doe knows the lottery numbers and will play them tomorrow, then he will win." and (ii) "Doe knows the lottery numbers and will play them tomorrow." is unintuitive only in the case that we forget about our commitment to (i) and (ii), since after all it is very unlikely for anybody to win the lottery.

Opposite to the premise-dependend treatment of conclusions in the case of classical logic, "the evaluation should [in the case of deontic detachment] be considered in a defeasible way.". This is also clear looking again at Example (G): naturally the antecedent of G1, $T$, is fulfilled. However the antecedent of the more specific G2 is also fulfilled, which leads in Wang's perspective to G1 being defeated.

Wang criticizes McNamara for giving a premise-independent interpretation of the conclusion for the Gentle Murderer, while "obligatory in the premise [G2] is interpreted in the evaluative sense, i.e. given that Doe does kill his mother, it is better that Doe kills her gently, McNamara objects to the conclusion by a moral sense of the obligation based on what is morally right or wrong."

Prakken/Sergot point out that the relationship between primary and secondary obligation in a CTD situation can best be modelled by regarding the secondary obligation as being relative to a 'context' where "a context stands for a constellation of acts or situations that agents regard as being settled in determining what they should do." ([14], p. 224) A "key difference between contrary-to-duty and prima facie obligations" is that, unlike prima facie obligations and opposite to Wang's view, contextual obligations in the sense of Prakken/Sergot do not satisfy any form of (defeasible) factual detachment. The obligation not to kill is not overridden in our case, it is fully valid. Furthermore, detaching the obligation to kill gently would

\footnotetext{
${ }^{5}$ Along these lines one might of course still object against the premise G2 itself instead of questioning the inference.
} 
cause an inconsistency which is "counter to our intuitions",

Torre/Tan share in [18] a similar intuition about such cases: In cases such as the asparagus example they speak of an obligation, e.g. $\mathrm{O}(\neg f \mid \top)$, being overridden and cancelled by a more specific obligation, e.g. $\mathrm{O}(f \mid a)$. However, in CTD situations they use the term overshadowing: for instance $\mathrm{O}(\neg k \mid T)$ is overshadowed by $\mathrm{O}(g \mid k)$ in a situation in which the killing takes place. The primary obligation, though being violated, is not in any way cancelled or destroyed. Therefore, they argue, it would be intuitive to derive $O \neg k$, i.e. to apply detachment to $\mathrm{O}(\neg k \mid \top)$, while "[t]he consistency of $O \neg k \wedge O g$ is a solution that seems like overkill." ([20], p. 53) With respect to our terminology it seems to be the intuition of Torre/Tan that in most cases excepted obligations are being overridden while violated obligations are being overshadowed. ${ }^{7}$ It can be argued that Torre/Tan circumvent the criticism by Wang: they evaluate the conclusion in a premisedependent way and it is precisely the nature of contrary-to-duty premises that justifies and motivates that detachment is not applied to them, but rather to the more general obligation which is being violated. The intuition is that whether Doe kills his mother or not, he has the obligation not to kill her and this obligation binds him. In the former case he violates this obligation.

However, one might ask, what kind of conditional G2 represents, since, although it is of the form "if ..., then ...", the logic does in no way allow to detach its conclusion. Nevertheless, in case of G3, G2 binds Doe in a certain way. This would with Tan/Torre's as well as with Prakken/Sergot's proposal not be represented by a deontic logic modelling detachment. In fact, both, G1 and G2 are of a binding nature in case of G3, despite the fact that both conclusions are incoherent. Thus, I suggest that a proper deontic logic allowing for detachment should be able to distinguish these cases and to offer an adequate detachment mechanism for both intuitions.

Instrumental and proper obligations So, who is right, Wang or McNamara, Prakken/Sergot and Tan/Torre? I would like to suggest: all of them in their own way. This is due to the fact that different intuitions are in place which are all justified in their own respect once they are made more transparent.

The way in which Wang is right can be clarified with what Foot dubs 'obligations of type 2' which "tell us the right thing to do" ([3], p. 385). They answer the question "And what all things considered ought we to do?" (p. 386). We call these obligations from now on instrumental obligations and we write $\mathrm{O}^{\mathrm{i}} A$ for the instrumental obligation to bring about $A$. They tell us modulo obligations we violated already, i.e. without considering already violated obligations, what we should and can bring about.

They seem to correspond to Wang's intuition. Interpreting the factual premises as settled and not being anymore avoidable, all-things-considered it is the best for Doe to kill his mother gently, presupposing that he kills her. Thus, violated obligations are not candidates for detaching instrumental obligations. Rather are the violating CTD-obligations the ones which should be detached: in this case to kill the mother gently. Also, in case a promise is broken the weak CTD-obligation to apologize should be considered as an instrumental obligation to be detached. Excepted obligations are no candidates for instrumental detachment: being served asparagus we are obviously exempted from the obligation to eat with fingers. It

\footnotetext{
${ }^{6}$ The only form of detachment contextual obligations satisfy is the following strong detachment principle: $\models(O[B] A \wedge \square B) \supset O A$, where $\square$ is the necessity operator of an adequate modal logic.

${ }^{7}$ However, Torre/Tan did not provide a precise definition, but rather an intuitive characterization of overshadowing. In Section $3 \mathrm{I}$ propose a formal definition for these notions. Furthermore in Footnote 15 I give an example for a violated obligation which is not overshadowed.
} 
has to be considered as being overridden and the more specific obligation to eat with fingers should be detached as instrumental obligation. 8

Note that instrumental obligations are both 'actual' (in our preliminary understanding of not being cancelled out or destroyed by another obligation) and they bind us in the sense of guiding our actions.

The intuition behind Prakken and Tan/Torre's approach on the other hand seems to be motivated by the aim to detach what is often called obligations proper, or actual, or absolute obligations. We call these obligations from now on proper obligations and write $\mathrm{O}^{\mathrm{p}} A$ for the proper obligation to bring about $A$. They tell us, all-things-considered, what our obligations in a certain situation are, also taking into account obligations which we might have violated already but which still bind us. That they are 'actual' despite being violated is the conditio that we judge a violator of being guilty and is being witnessed by us having a bad conscience once having violated them.

In the case of Example (G), all-things-considered the moral reasons for not killing his mother are stronger than the reasons for killing her gently: after all, the obligation to kill her gently is only secondary for the case that he violates the primary obligation. It provides a practical guideline how to act in the situation in which the murder takes place, however the obligation proper for Doe is not to kill. 9 Thus, we are interested in detaching violated obligations rather than their (strong) CTD-violaters. This is a key difference to the instrumental case. However, weak CTD-obligations should be detached as being proper obligations since all-thingsconsidered we are obliged to apologize in case we break a promise and this obligation is not in conflict with any more general obligation that would, all-things-considered, bind us more. Analogously to the instrumental case, excepted oblgations should not be detached, but rather the more specific obligation which overrides them: it is our proper obligation to eat with fingers in case asparagus is served 10

Concluding it can be said that proper obligations bind us even in the case that they are violated by secondary obligations. They are 'actual' in our preliminary understanding since, also in the case of being violated they are not destroyed or cancelled, or as Tan/Torre put it: they are merely overshadowed.

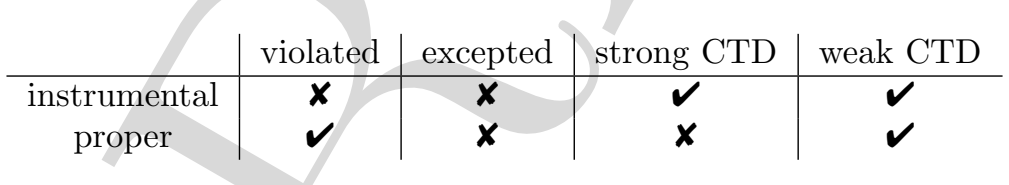

Table 1: What is detached? The columns are considered to be mutually exclusive.

In an 'ideal world' in which no obligation is violated, both concepts, instrumental and proper obligations, coincide. This might very well be a world in which we eat asparagus or in which other exceptional contexts with respect to some conditional obligations are valid. As discussed, exceptional contexts do not cause obligations to be violated, although they are excepted. In terms of Torre/Tan, 'ideal worlds' in this sense are such that no overshadowing takes place. However,

\footnotetext{
${ }^{8} \mathrm{Cp}$. Table 1 for an overview.

${ }^{9}$ Some dialethists might, opposite to McNamara and Torre/Tan, argue that both, the secondary and the primary obligation, are proper obligations in the violation context, despite the fact that they contradict each other. I do not wish to speak the final word on this. It should however be noted that the dialethist might want to interpret $\mathrm{O}^{\mathrm{P}} A$ as: (i) the obligation to bring about $A$ is a proper obligation and (ii) the obligation to bring about $A$ is stronger than all other proper obligations incoherent with $A$. I take it for granted that dialethists would rather turn back the wheel of time in case Doe kills his mother than letting him kill her gently, thus the primary obligation $\mathrm{O}(\neg k \mid \mathrm{T})$ should be considered to be stronger than the secondary $\mathrm{O}(g \mid k)$.

${ }^{10} \mathrm{Cp}$. Table 1 for an overview.
} 
as soon as overshadowing enters the scene, our instrumental obligations begin to differ from our proper obligations, as we have seen for instance with the Gentle Murderer example. Proper obligations have been violated, but they are still valid.

\begin{tabular}{l|c|c|c|c} 
Example & $(\mathrm{A})$ & $(\mathrm{G})$ & $(\mathrm{Gx})$ & $(\mathrm{PA})$ \\
\hline Premises & $\mathrm{O}(\neg f \mid \mathrm{T})$ & $\mathrm{O}(\neg k \mid \mathrm{T})$ & $\mathrm{O}(\neg k \mid \mathrm{T})$ & $\mathrm{O}(\neg b \mid \mathrm{T})$ \\
& $\mathrm{O}(f \mid a)$ & $\mathrm{O}(g \mid k)$ & $\mathrm{O}(g \mid k)$ & $\mathrm{O}(a \mid b)$ \\
& $a$ & & $\mathrm{P}(k \mid x)$ & \\
& & $k$ & $x$ & $\neg k$ \\
\hline proper & $\mathrm{O}^{\mathrm{p}} f$ & $\mathrm{O}^{\mathrm{p}} \neg k$ & $\mathrm{O}^{\mathrm{p}} g$ & $\mathrm{O}^{\mathrm{p}} \neg b, \mathrm{O}^{\mathrm{p}} a$ \\
instrumental & $\mathrm{O}^{\mathrm{i}} f$ & $\mathrm{O}^{\mathrm{i}} g$ & $\mathrm{O}^{\mathrm{i}} g$ & $\mathrm{O}^{\mathrm{i}} a$ \\
\hline context & exceptional & violation & exceptional & violation \\
\hline over... & overriding & overshadowing & overriding & overshadowing \\
\hline relationship & specifity & CTD (strong) & specifity & CTD (weak)
\end{tabular}

Table 2: Overview of the Examples

Note that in the case of the asparagus example proper and instrumental obligations coincide, $\mathrm{O}^{\mathrm{p}} f$ and $\mathrm{O}^{\mathrm{i}} f$, since $\mathrm{O}(\neg f \mid \mathrm{T})$ has been excepted. It is also interesting to contrast the weak CTD in our (PA) example with the Gentle Murderer: the secondary obligation to apologize is coherent with the primary obligation not to break a promise. Analogous to the Gentle Murderer the primary obligation gives us a proper obligation, i.e. $\mathrm{O}^{\mathrm{p}} \neg b\left(\right.$ resp. $\mathrm{O}^{\mathrm{p}} \neg k$ ), while the secondary obligation provides us with a instrumental obligation, i.e. O' $a$ (resp. $\mathrm{O}^{\mathrm{i}} g$ ). However, it also provides us with a proper obligation, $\mathrm{O}^{\mathrm{P}} a$ (but not $\left.\mathrm{O}^{\mathrm{P}} \mathrm{g}\right) .11$

Let us close this section with another instructive example (Gx): Read for instance $x$ as "Doe's mother is a tyrant who is about to do something very evil (such as activating an atom bomb, etc.) and the only way to stop her in a certain situation is to kill her". From the perspective of some moral standards it might be argued that in this situation Doe is, if not obliged to kill his mother, so at least allowed to kill her, $\mathrm{P}(k \mid x)$. Note that $x$ describes an exceptional context, while $k$ alone describes a violation context with respect to the primary obligation $\mathrm{O}(\neg k \mid \top)$. Thus, in the case $x$ or $x \wedge k, \mathrm{O}(\neg k \mid \top)$ is excepted and not violated. Hence, as discussed above, neither proper nor instrumental detachment should be applied to it.

The example is also instructive in another respect: Due to the fact that $\mathrm{O}(\neg k \mid$ $\top)$ is overridden by $\mathrm{P}(k \mid x)$ and $x$, the question arises again whether factual detachment should be applied to $\mathrm{O}(g \mid k)$ or $\mathrm{O}(g \mid k \wedge x)$ in case of $x \wedge k$. Here our intuition is, despite $\mathrm{O}(g \mid k)$ being a (strong) CTD-obligation, that we want to apply factual detachment, not just in the instrumental case, but also in order to detach it as a proper obligation. This is due to the fact that the corresponding primary obligation, $\mathrm{O}(\neg k \mid \mathrm{T})$, is excepted.

\section{Formally realizing detachment}

For the formal part we presuppose a propositional calculus which we supplement by a dyadic obligation operator $\mathrm{O}$ for conditional obligations $\mathrm{O}(A \mid B)$ where $A$ and

\footnotetext{
${ }^{11}$ Of course, in an 'ideal' world there is no need to apologize, thus, the fact that in the case of $b$ both obligations, to keep the promise and to apologize, are 'actual', Prakken/Sergot in [13] call a 'pragmatic oddity'. Tan/Torre ([20], p. 63) call it counter-intuitive. Taking into account however that (a) some proper obligations may be violated while other proper obligations might still be realizable (the ones which are also instrumental obligations such as in our case the obligation to apologize) and (b) that the two obligations in question are coherent, I do not think this is counter-intuitive.
} 
$B$ are propositional formulas and analogously $\mathrm{P}$ for permissions, ${ }^{12}$ Furthermore we use two monadic obligation operators, $\mathrm{O}^{\mathrm{p}}$ and $\mathrm{O}^{\mathrm{i}}$, for proper and instrumental obligations. Where $\wedge, \vee, \supset$ and $\neg$ are the classical logical operators, we add two further, paraconsistent negations: $\stackrel{p}{\neg}$ and $\stackrel{i}{\neg}$. The negations are characterized by the law of excluded middle (LEM), $A \wedge \sim A$ where $\sim \in\{\stackrel{\mathrm{p}}{\neg}, \stackrel{\mathrm{i}}{\neg}\}$. The intended meaning of ${ }^{\mathrm{p}} \mathrm{O}(A \mid B)$ (resp. ${ }_{\neg}^{\mathrm{i}} \mathrm{O}(A \mid B)$ ) is that the commitment to bring about $A$ if $B$, is blocked from being detached as a proper (resp. instrumental) obligation. We therefore alter the rule for factual detachment $(\overline{F D})$ as follows:

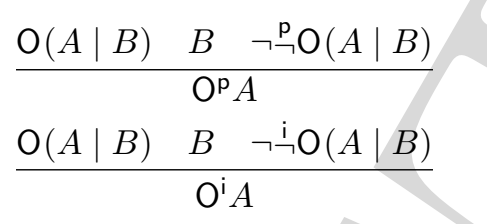

We restrict our language in such a way that $\stackrel{p}{\neg}$ and $\stackrel{i}{\neg}$ only precede conditional obligations. Note that the following is an immediate consequence of (LEM): $\neg \mathrm{O}(A \mid B) \supset \stackrel{\mathrm{x}}{\mathrm{O}}(A \mid B)$ where $\mathrm{x} \in\{\mathrm{i}, \mathrm{p}\}$. In order to make use of these rules we have to define rules which govern the overriding and overshadowing of conditional obligations.

Blocking proper detachment The following rule realizes specifity as discussed in Section 2: excepted obligations are not to be detached as proper obligations. ${ }^{13}$

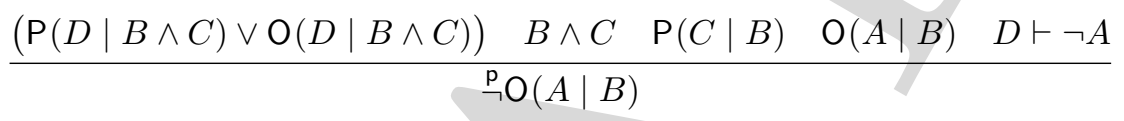

Note that in our asparagus example, $-\mathrm{O}(\neg f \mid \top), \mathrm{O}(f \mid a), \mathrm{P}(a \mid \top)$ - we derive ${ }^{\mathrm{P}} \mathrm{O}(\neg f \mid \mathrm{T})$ from the given premises, whenever $a$ is the case.

We are furthermore interested in blocking proper detachment in (strong) CTD cases. While in the case of specifity we block the more general primary obligation, the situation is now inverse:

$$
\frac{\mathrm{O}(A \mid B \wedge C) \quad \mathrm{O}(D \mid B) \quad D \vdash \neg(B \wedge C) \quad D \vdash \neg A}{{ }_{\neg}^{\mathrm{P}}(A \mid B \wedge C)}
$$

In the case of the Gentle Murderer we thus derive ${ }^{\mathrm{p}} \mathrm{O}(g \mid k)$ from $\mathrm{O}(\neg k \mid \top)$.

Blocking instrumental detachment As discussed above, we intend to evaluate the premises as facts which are not anymore alterable and thus if $\neg A$ is the case we avoid instrumental obligations such as $\mathrm{O}^{\mathrm{i}} A$, since conditional obligations with conclusion $A$ are de facto violated:

$$
\frac{\mathrm{O}(A \mid B) \neg A \quad B}{\neg \mathrm{O}(A \mid B)}
$$

For instance in the Gentle Murderer case (G) we have $k$ and thus we get $\neg_{\neg} \mathrm{O}(\neg k \mid \top)$. Similarly we get in Chisholm's example (C) $\stackrel{\mathrm{i} O}{\neg}(\neg i \mid \top)$ since we have $i$ where $i$ stands for 'John impregnates Diane'.

Furthermore, we prioritize more specific cases over incoherent general obligations: ${ }^{13,14}$

$$
\frac{(\mathrm{P}(D \mid B \wedge C) \vee \mathrm{O}(D \mid B \wedge C)) \quad B \wedge C \quad \mathrm{O}(A \mid B) \quad D \vdash \neg A}{\stackrel{\mathrm{i} O}{ } \mathrm{O}(A \mid B)}
$$

\footnotetext{
${ }^{12}$ The permission operator may also be defined as $\mathrm{P}(A \mid B)=_{\mathrm{df}} \neg \mathrm{O}(\neg A \mid B)$.

${ }^{13}$ In logics verifying $\vdash \mathrm{O}(A \mid B) \supset \mathrm{P}(A \mid B)$ (e.g. standard deontic logic) the first condition of the antecedent can be simplified to $\mathrm{P}(\neg A \mid B \wedge C)$.

${ }^{14}$ This is a heuristic principle used in this paper.
} 
Note that if the antecedent of rule (oV-Ei) is valid, then either $\mathrm{O}(A \mid B)$ is excepted (in case $\mathrm{P}(C \mid B)$ is valid), or $\mathrm{O}(A \mid B)$ is violated. As discussed in Section 2, in both cases we do not want to $\operatorname{detach} \mathrm{O}^{\mathrm{i}} A$.

For instance in the asparagus case we get $\stackrel{\mathrm{i}}{\mathrm{O}}(\neg f \mid \top)$ due to $a$ and $\mathrm{O}(f \mid a)$.

Overriding and overshadowing With our terminology we are able to propose a precise characterization of overriding and overshadowing: we say that an obligation $\mathrm{O}(A \mid B)$ is overshadowed iff $\neg \mathrm{i}(A \mid B)$ is derivable and ${ }^{\mathrm{p}} \mathrm{O}(A \mid B)$ is not derivable. We say that $\mathrm{O}(A \mid B)$ is overridden iff ${ }_{\neg}^{\mathrm{P}} \mathrm{O}(A \mid B)$ and ${ }_{\neg}^{\mathrm{i}} \mathrm{O}(A \mid B)$ are derivable or $\neg B$ is derivable. It is said to be overridden by specifity in the former case, while it is said to be factually overridden in case $\neg B$ is derivable ${ }^{15}$

Realizing detachment by an adaptive logic Extending a given dyadic deontic logic $\mathbf{L}$ by the new rules stated above resulting in $\mathbf{L}^{+}$does not yet give a satisfactory account of detachment. In order to show this we take a look at two paradigmatic proofs of example (A) (to the left) and example (G) (to the right):

\begin{tabular}{|c|c|c|}
\hline & $\mathrm{O}(\neg f \mid \top)$ & PREM \\
\hline & $\mathrm{O}(f \mid a)$ & PREM \\
\hline & $\mathrm{P}(a \mid \mathrm{\top})$ & PREM \\
\hline & $a$ & PREM \\
\hline & $\mathrm{i} \mathrm{O}(\neg f \mid \top)$ & $1,2 \sqrt{4}$ \\
\hline & $\stackrel{\mathrm{p}}{\neg} \mathrm{O}(\neg f \mid \mathrm{T})$ & $1,2,:$ \\
\hline
\end{tabular}

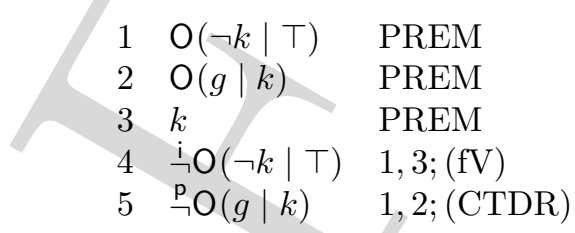

Although the logic is able to block undesired applications of the factual detachment rules (FDi) and (FDp), we are not able to apply them where needed. For instance in the case of the asparagus example (A) we are interested in applying (FDi) and (FDp) to $\mathrm{O}(f \mid a)$, in the case of the Gentle Murderer we are interested in applying (FDi) to $\mathrm{O}(g \mid k)$ and $(\mathrm{FDp})$ to $\mathrm{O}(\neg k \mid \mathrm{T})$. What we are missing are means to derive $\neg \neg \mathfrak{\mathrm { i }} \mathrm{O}(f \mid a)$ and $\neg \neg \mathrm{p} \mathrm{O}(f \mid a)$ in the former case and $\neg \stackrel{\mathrm{i}}{\neg} \mathrm{O}(g \mid k)$ and $\neg \stackrel{\mathrm{p}}{\mathrm{O}}(\neg k \mid \top)$ in the latter case.

This is where the adaptive logic comes in since these logics allow for conditional applications of certain rules which enable them to interpret a premise set "as normally as possible" with respect to some given criterion for normality. In our case we are interested in applying (FDi) (resp. (FDp) to $\mathrm{O}(A \mid B)$ and $B$ on the condition that $\stackrel{\mathrm{i}}{\neg} \mathrm{O}(A \mid B)$ (resp. $\left.{ }^{\mathrm{p}} \mathrm{O}(A \mid B)\right)$ is not derivable. For instance in the Gentle Murderer example it would be useful to extend the proof by the following lines: 16

$$
\begin{array}{llll}
7 & \mathrm{O}^{\mathrm{i} g} & 2,3 ;(\overline{\mathrm{FDi}})^{c} & \left\{\mathrm{O}(g \mid k) \wedge \wedge^{\mathrm{i}} \mathrm{O}(g \mid k)\right\} \\
8 & \mathrm{O}^{\mathrm{P}} \neg k & 1 ;\left(\mathrm{FDp}^{c}\right. & \left\{\mathrm{O}(\neg k \mid \mathrm{T}) \wedge{ }^{\mathrm{p}} \mathrm{O}(\neg k \mid \mathrm{T})\right\}
\end{array}
$$

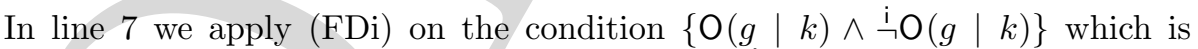
written in the last column. The idea is, that in case ${ }^{\mathrm{i}} \mathrm{O}(g \mid k)$ is derived at a later stage of the proof, we mark all lines that have $\mathrm{O}(g \mid k) \wedge \neg \mathrm{O}(g \mid k)$ as an element of the condition. The second element of such lines are not anymore considered to be derivable.

Adaptive Logics The mechanism of adaptive logics has been presented in various papers. The space limitations require that we refer the reader interested in a detailed description of them to [1]. Here we will only mention some key features.

\footnotetext{
15 Note that this is not a correct characterization of violated and excepted obligations. Say we have $\mathrm{O}(\neg k \mid \mathrm{T})$ and $\mathrm{O}(g \mid k)$ as in (G) and additionally $\neg g$ : $\mathrm{O}(g \mid k)$ is a violated (strong) CTD-obligation. However, it is not overshadowed and we have $\neg \mathrm{O}(g \mid k)$ and ${ }^{\mathrm{p}} \mathrm{O}(g \mid k)$.

${ }^{16} \mathrm{We}$ write for instance $(\mathrm{FDi})^{c}$ in the third column of the proof in order to indicate that (FDi) is applied conditionally.
} 
An adaptive logic in standard format is a triple consisting of (i) a lower limit logic (henceforth LLL), which is a reflexive, transitive, monotonic, and compact logic that has a characteristic semantics and contains CL (classical logic), (ii) a set of abnormalities $\Omega$, characterized by a (possibly restricted) logical form, and (iii) an adaptive strategy. Formulating an adaptive logic in the standard format provides the logic with all of the important meta-theoretic features, such as soundness and completeness (as is shown in [1]).

In the following we use $\varphi$ and $\psi$ as meta-variables for well-formed formulas of a given language. The proof dynamics is governed by marking conditions for proof lines. The fact that the proofs are of a dynamic nature makes adaptive logics very useful for the modeling of defeasible reasoning, since a formula derivable at one stage of the proof may turn out to be underivable at a later stage. A line of a proof consists of a line number, a formula, a justification, and a condition. Conditions are finite subsets of the set of abnormalities. We abbreviate $\bigvee_{\varphi \in \Delta} \varphi$ with $\operatorname{Dab}(\Delta)$ for some finite set $\Delta$ of abnormalities. All the rules of the LLL are valid. The conditions in the fourth column of the proof are carried forward, i.e. if $\varphi_{1}, \ldots, \varphi_{n} \vdash_{\text {LLL }} \psi$ and the $\varphi_{i}$ 's were derived on the conditions $\Delta_{i}$, then the adaptive logic allows to derive $\psi$ on the condition $\Delta_{1} \cup \cdots \cup \Delta_{n}$. Furthermore, if the following is valid in the $\mathbf{L L L}, \varphi_{1}, \ldots, \varphi_{n} \vdash_{\mathbf{L L L}} \psi \vee \operatorname{Dab}(\Theta)$ and the $\varphi_{i}$ 's were derived on conditions $\Delta_{i}$, then the adaptive logic derives $\psi$ on condition $\Delta_{1} \cup \cdots \cup \Delta_{n} \cup \Phi$.

In order to define the strategy used in this paper some more terminology has to be introduced. $\operatorname{Dab}(\Delta)$ is a minimal Dab-formula at a stage $s$ of the proof iff it is the formula of a line with condition $\emptyset$ and no $\operatorname{Dab}\left(\Delta^{\prime}\right)$ with $\Delta^{\prime} \subset \Delta$ is the formula of a line with condition $\emptyset$. We call the minimal Dab-formulas derivable with the LLL, the minimal Dab-consequences. A choice set of $\Sigma=\left\{\Delta_{1}, \Delta_{2}, \ldots\right\}$ is a set that contains one element out of each member of $\Sigma$. A minimal choice set of $\Sigma$ is a choice set of $\Sigma$ of which no proper subset is a choice set of $\Sigma$. Where, for a premise set $\Gamma, \operatorname{Dab}\left(\Delta_{1}\right), \ldots, \operatorname{Dab}\left(\Delta_{n}\right)$ are the minimal Dab-formulas at stage $s$, $\Phi_{s}(\Gamma)$ is the set of minimal choice sets of $\left\{\Delta_{1}, \ldots, \Delta_{n}\right\}$.

Definition 1 (Marking for minimal abnormality). Line $i$ is marked at stage $s$ iff, where $\varphi$ is derived on the condition $\Delta$ at line $i$,

(i) there is no $\Delta^{\prime} \in \Phi_{s}(\Gamma)$ such that $\Delta^{\prime} \cap \Delta=\emptyset$, or

(ii) for some $\Delta^{\prime} \in \Phi_{s}(\Gamma)$, there is no line at which $\varphi$ is derived on a condition $\Theta$ for which $\Delta^{\prime} \cap \Theta=\emptyset$.

Note that a line might be marked at stage $s$ of the proof, but become unmarked at a later stage $s+i$. Indeed, even if $\operatorname{Dab}(\Delta)$ is a minimal Dab-formula at stage $s$, we might be able to derive $\operatorname{Dab}\left(\Delta^{\prime}\right)$ where $\Delta^{\prime} \subset \Delta$ at stage $s+i$ which may lead to an alteration of the minimal choice sets and thus to changes in the marking of lines. Given a set of abnormalities $\Omega$, it is the job of the marking conditions to determine if lines are "in" or "out" of the proof at a certain stage, i.e. to govern the internal dynamics of the proof procedure.

In order to define the consequence set of an adaptive logic we are interested in a stable criterion for derivability.

Definition 2. $A$ is finally derived from $\Gamma$ on line $i$ of a proof at stage $s$ iff (i) $\varphi$ is the second element of line $i$, (ii) line $i$ is not marked at stage $s$ and (iii) every extension of the proof in which line $i$ is marked may be further extended in such a way that line $i$ is unmarked.

$\Gamma \vdash_{\mathbf{A L}} \varphi$ ( $\varphi$ is finally $\mathbf{A L}$-derivable from $\Gamma$ ) iff $\varphi$ is finally derived on a line of a proof from $\Gamma$. 
Let's have a look at the semantics: The idea behind the minimal abnormality strategy is that only the models (of a given premise set) which validate a minimal set of abnormalities (that is, which are the "minimal abnormal" ones w.r.t. $\subset$ ) are taken into account.

Definition 3. $\Gamma \models_{\text {AL }} \varphi$ ( $\varphi$ is an AL-semantic consequence of $\Gamma$ ) iff $\varphi$ is verified by all minimally abnormal LLL-models of $\Gamma$. A LLL-model $M$ of $\Gamma$ is minimally abnormal iff there is no LLL-model $M^{\prime}$ of $\Gamma$ such that $\mathrm{Ab}\left(M^{\prime}\right) \subset \mathrm{Ab}(M)$.

Note furthermore that the following completeness and soundness result is valid for all adaptive logics $\mathbf{A L}$ in standard format: $\Gamma \vdash_{\mathbf{A L}} \varphi$ iff $\Gamma \models_{\mathbf{A L}} \varphi$.

A generic adaptive logic for detachment Given our logic $\mathbf{L}^{+}$satisfies the criteria for lower limit logics stated above, we are able to define an adaptive logic $\mathbf{D L}^{+}$which models detachment as follows:

- the $\mathbf{L L L}$ is $\mathbf{L}^{+}$

- the abnormalities are $\Omega^{d}=_{\mathrm{df}} \Omega^{\mathrm{i}} \cup \Omega^{\mathrm{p}}$ where $\Omega^{\mathrm{x}}={ }_{\mathrm{df}}\{\mathrm{O}(A \mid B) \wedge \stackrel{\times}{\neg} \mathrm{O}(A \mid B)$ : $A, B \in \mathcal{P}\}$ for $\mathrm{x} \in\{\mathrm{i}, \mathrm{p}\}$ and $\mathcal{P}$ being the set of all propositional formulas;

- the strategy is minimal abnormality.

In the remainder of the paper we demonstrate this by using a variant of Lou Goble's CDPM as lower limit.

\section{A lower limit: Lou Goble's CDPM}

In order to demonstrate the adaptive modelling of detachment by means of a concrete logic we settle for this paper on a variant of Lou Goble's CDPM as lower limit logic. This choice is not essential for our approach for handling detachment by means of an adaptive logic, it is possible to use other logics as lower limit. The choice however is not arbitrary either since CDPM has many nice properties. For instance it tolerates deontic conflicts while blocking unintuitive consequences from conflicting obligations, ${ }^{17}$ Furthermore, in the absence of deontic conflicts it offers a close approximation of (dyadic) standard deontic logic 18 One of the main ideas behind this logic is to restrict the inheritance principle

$$
\text { If } \vdash B \supset C \text { then } \vdash \mathrm{O}(B \mid A) \supset \mathrm{O}(C \mid A)
$$

by adding a further permission statement:

$$
\text { If } \vdash B \supset C \text { then } \vdash \mathrm{P}(B \mid A) \supset(\mathrm{O}(B \mid A) \supset \mathrm{O}(C \mid A))
$$

This principle is not less intuitive then (CRM) and harmless from the perspective of standard deontic logic, since there $\mathrm{O}(A \mid B) \vdash \mathrm{P}(A \mid B)$ is valid and therefore also the equivalence of (CRM) and ( $\mathrm{RCPM})$. However, this principle ensures that the logic is non-explosive confronted with deontic conflicts.

Lou Goble proposed in [7] two versions of his monadic DPM systems: DPM.1 and DPM. 2 where only the latter verifies the rule $(\mathrm{P}), \vdash \neg \mathrm{O} \perp$. In order to ensure non-explosive behaviour in DPM.2 the aggregation principle had to be restricted

\footnotetext{
${ }^{17}$ Neither of the following "deontic explosion principles" is valid in any of the CDPM versions which are going to be introduced in this paper:

$$
\begin{aligned}
& \text { If } \nvdash B \text { then } \vdash(O A \wedge O \neg A) \supset O B \\
& \vdash(O A \wedge O \neg A) \supset(P B \supset O B) \\
& \vdash(O C \wedge P C) \supset((O A \wedge O \neg A) \supset(P B \supset O B))
\end{aligned}
$$

${ }^{18}$ Lou Goble defines a dyadic version SDDL of standard deontic logic in [6]. It is equivalent to van Fraassen's CD of [21] and David Lewis' VN of [10].
} 
in a similar way as inheritance. For the conditional version Goble favours the second version, since in the first one an unintuitive kind of strengthening of the antecedent (SA) is valid. 19 We thus focus for this paper on the system Goble dubs CDPM.2c. One of the characteristic features of this logic is its handling of (SA). Goble gives an account of $(\mathrm{SA})$ by restricting rational monotonicity: 20

$$
\vdash(\mathrm{O}(B \mid A) \wedge \mathrm{P}(B \wedge C \mid A)) \supset \mathrm{O}(B \mid A \wedge C)
$$

CDPM.2c is defined by adding to all instances of ( $\mathrm{RCPM})$ and (WRM) all instances of the following axioms:

$$
\begin{array}{r}
\vdash \neg \mathrm{O}(\perp \mid A) \\
\text { If } \vdash A \equiv B \text { then } \vdash \mathrm{O}(C \mid A) \equiv \mathrm{O}(C \mid B) \\
\text { If } \vdash A \equiv B \text { then } \vdash \mathrm{O}(B \mid A) \equiv \mathrm{O}(C \mid A) \\
\vdash \mathrm{O}(\top \mid \top) \\
\vdash \mathrm{O}(B \mid A) \supset \mathrm{O}(A \mid A) \\
\vdash \mathrm{O}(C \mid A \wedge B) \supset \mathrm{O}(B \supset C \mid A) \\
\vdash(\mathrm{O}(A \mid C) \wedge \mathrm{O}(B \mid C) \wedge \mathrm{P}(A \wedge B \mid C)) \supset \mathrm{O}(A \wedge B \mid C)
\end{array}
$$

Axiom (QR) is in some cases severely counter-intuitive: take the Chisholm example (C) from page 2. It allows to derive from the commitment to marry Diane in case John impregnates her, the commitment to impregnate her in that very case. However, the fact that he impregnates her is a violation of the primary obligation not to impregnate her. We therefore abandon rule $(\mathrm{QR}) \cdot{ }^{21}$ Furthermore, we extend our language as discussed in Section 3 and add rules (FDp), (FDi), (Ep), (CTDR), (fV) and (oV-Ei). The new logic is dubbed CDPM.2d. For reasons which are explicated later (cp. page 14) we present an alternative version of our logic by replacing $(\mathrm{S})$ with the following restricted version

$$
\vdash(\mathrm{O}(A \mid B \wedge C) \wedge \mathrm{P}(A \mid \neg B \wedge C)) \supset \mathrm{O}(B \supset A \mid C)
$$

We dub this logic CDPM.2e. Both logics, CDPM.2d and CDPM.2e serve as candidates for our lower limit logics.

The semantics for these systems can be found in the Appendix.

\section{$5 \quad$ Handling detachment adaptively}

As already explicated at the end of Section 3, the idea is to apply factual detachment to $\mathrm{O}(A \mid B)$ and $B$ for proper (resp. instrumental) obligations on the condition that $\stackrel{\mathrm{p}}{\neg} \mathrm{O}(A \mid B)$ (resp. $\stackrel{\mathrm{i}}{\neg} \mathrm{O}(A \mid B))$ is not derivable. This is realized by the following adaptive logic:

\footnotetext{
${ }^{19}$ It validates all instances of $\vdash(\mathrm{O}(B \mid A) \wedge \mathrm{P}(C \mid A) \wedge \mathrm{P}(B \mid A)) \supset \mathrm{O}(B \mid A \wedge C)$ (PRatMono). That (PRatMono) is counter-intuitive can be demonstrated by means of our asparagus example: if we add the intuitive premise $\mathrm{P}(\neg f \mid \top)$, then the counter-intuitive $\mathrm{O}(\neg f \mid a)$ is derivable. This defect is not fatal though: I proposed in [16] a version of CDPM.1 which overcomes this shortcoming.

${ }^{20}$ This principle (cp. [9]) can be stated in terms of the language for dyadic deontic used in this paper as follows: $\vdash(\mathrm{O}(B \mid A) \wedge \mathrm{P}(C \mid A)) \supset \mathrm{O}(B \mid A \wedge C)$. It is verified in dyadic standard deontic logic. Goble is aware of the fact that his (WRM) leads to counter-intuitive, even explosive behaviour in some cases (for instance take our asparagus example and replace $\mathrm{O}(f \mid a)$ by $\mathrm{P}(f \mid a)$ and add $\mathrm{P}(\neg f \wedge a \mid \mathrm{T}))$. I offered an improvement based on the idea of conditionally applying (SA) within an adaptive logic which is able to avoid these problems (cp. Section 6).

${ }^{21}$ The reader might further object that (CN) (in a similar way as (QR)) is not very intuitive or give philosophically minded reasons why we should abandon it as well. Goble's intention is to stay as close as possible to standard deontic logic. However, (CN) is neither an essential part of his logic nor in any way essential to our approach and may thus be disregarded as well.
} 
Definition 4. DCDPM $\alpha$ where $\alpha \in\{\mathbf{d}, \mathbf{e}\}$ is defined as an adaptive logic in standard format by the following triple:

- the lower limit logic is CDPM.2 $\alpha$;

- the set of abnormalities is $\Omega^{d}$

- the strategy is minimal abnormality.

\subsection{Some examples}

Let us demonstrate the way the logic works by having a look at some examples.

Example (G cp. page 10). We already stated a proof for the case of the Gentle Murderer on page $10 \sqrt{22}$ Note that there is no way to extend the proof in such a way that lines 7 and 8 are marked, i.e. there is no way to derive ${ }_{-} \mathrm{O}(g \mid k)$ and ${ }^{\mathrm{P}} \mathrm{O}(\neg k \mid \mathrm{T})$ as part of minimal Dab-consequences in our lower limit CDPM. $2 \alpha$ $(\alpha \in\{\mathbf{d}, \mathbf{e}\})$. Therefore, $\mathrm{O}^{\mathrm{i}} g$ and $\mathrm{O}^{\mathrm{p}} \neg k$ are finally derivable.

Example (PA cp. page 4). The following proof is valid for both adaptive logics:

\begin{tabular}{|c|c|c|c|}
\hline 1 & $\mathrm{O}(\neg b \mid \top)$ & PREM & $\emptyset$ \\
\hline 2 & $\mathrm{O}(a \mid b)$ & PREM & $\emptyset$ \\
\hline 3 & $b$ & PREM & 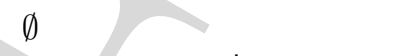 \\
\hline $5_{4}$ & $\mathrm{O}^{\mathrm{i}} \neg b$ & 1: $(\overline{\mathrm{FDi}})^{c}$ & )$\wedge \stackrel{\mathrm{i}}{\neg} \mathrm{O}(\neg b \mid \top)\}$ \\
\hline 5 & $\mathrm{O}(\neg b \mid \top) \wedge \stackrel{\mathrm{i}}{ } \mathrm{O}(\neg b \mid \top)$ & 1,$3 ;(\mathrm{fV})$ & $\emptyset$ \\
\hline 6 & $\mathrm{O}^{\mathrm{i}} a$ & $2 \sqrt{3} ;(\overrightarrow{\mathrm{FDi}})^{c}$ & $\{\mathrm{O}(a \mid b) \wedge \neg \mathrm{O}(a \mid b)\}$ \\
\hline 7 & $\mathrm{O}^{\mathrm{p}} a$ & 2,$3 ;(\overline{\mathrm{FDp}})^{c}$ & $\{\mathrm{O}(a \mid b) \wedge \stackrel{\mathrm{P}}{\neg} \mathrm{O}(a \mid b)\}$ \\
\hline 8 & $\mathrm{O}^{\mathrm{p}} \neg b$ & $1:(\mathrm{FDp})^{c}$ & $\{\mathrm{O}(\neg b \mid \top) \wedge \neg \mathrm{P}(\neg b \mid \top)\}$ \\
\hline
\end{tabular}

At lines 4, 6-8 we have conditional applications of factual detachment. Note that the first one gets marked, since its condition, $\mathrm{O}(\neg b \mid \mathrm{T}) \wedge \neg \mathrm{I} \mathrm{O}(\neg b \mid \mathrm{T})$, is derived on the empty condition at line 5. The other ones however are finally derivable.

\subsection{Deontic detachment and two intuitions concerning proper obliga- tions}

I postponed a discussion why rule (QR) was abandoned and why rule (S) was restricted to (PS') for CDPM.2e. Taking a look at the Chisholm example will illuminate these points: let $i$ stand for John impregnating Diane, and $m$ for him marrying her. We first take a look at a proof for DCDPMd:

\begin{tabular}{|c|c|c|c|}
\hline 1 & $\mathrm{O}(\neg i \mid \top)$ & PREM & $\emptyset$ \\
\hline 2 & $\mathrm{O}(\neg m \mid \neg i)$ & PREM & $\emptyset$ \\
\hline 3 & $\mathrm{O}(m \mid i)$ & PREM & $\emptyset$ \\
\hline 4 & $i$ & PREM & $\emptyset$ \\
\hline 5 & $\neg \mathrm{O}(\neg i \mid \mathrm{T})$ & 1,$4 ;(\mathrm{fV})$ & $\emptyset$ \\
\hline 6 & $\mathrm{O}(\neg i \supset \neg m \mid \top)$ & $2 ;(\mathrm{S})$ & $\emptyset$ \\
\hline 7 & $\mathrm{P}(\neg i \wedge \neg m \mid \top)$ & PREM & $\emptyset$ \\
\hline 8 & $\mathrm{O}(\neg i \wedge \neg m \mid \mathrm{\top})$ & $1,6,7 ;$ (CPAND) & $\emptyset$ \\
\hline 9 & $\mathrm{O}(\neg m \mid \top)$ & 7,$8 ;(\mathrm{RCPM})$ & $\emptyset$ \\
\hline 10 & ${ }_{7}^{\mathrm{p}} \mathrm{O}(m \mid i)$ & 3, $8,(\mathrm{CTDR})$ & $\emptyset$ \\
\hline 11 & $\mathrm{i} \mathrm{O}(\neg m \mid \mathrm{T})$ & $3,4,9 ;(\mathrm{oV}-\mathrm{Ei})$ & $\emptyset$ \\
\hline 12 & $\mathrm{O}^{\mathrm{i}} \mathrm{m}$ & 3,$4 ;(\overline{\mathrm{FDi}})^{c}$ & $\{\mathrm{O}(m \mid i) \wedge \stackrel{\mathrm{i}}{\sim} \mathrm{O}(m \mid i)\}$ \\
\hline 13 & $\mathrm{O}^{\mathrm{p}} \neg i$ & 1; $(\overline{\mathrm{FDp}})^{c}$ & $\left\{\mathrm{O}(\neg i \mid \mathrm{T}) \wedge{ }^{\mathrm{p}} \mathrm{O}(\neg i \mid \mathrm{T})\right\}$ \\
\hline 14 & $\mathrm{O}^{\mathrm{p}} \neg m$ & 9; $(\overline{\mathrm{FDp}})^{c}$ & $\{\mathrm{O}(\neg m \mid \top) \wedge \stackrel{\mathrm{p}}{\neg} \mathrm{O}(\neg m \mid \mathrm{\top})\}$ \\
\hline
\end{tabular}

\footnotetext{
${ }^{22}$ Lines 145 are not stated in the form of an adaptive logic proof. It can be easily adjusted though by simply adding the empty condition $\emptyset$ as a fourth column.
} 
What follows is a continuation of the proof from line 5 on in DCDPMe:

$$
\begin{array}{llll}
6 & \mathrm{O}^{\mathrm{i}} m & 3 \sqrt{4 ;}(\overline{\mathrm{FDi}})^{c} & \left\{\mathrm{O}(m \mid i) \wedge{ }^{\mathrm{i}} \mathrm{O}(m \mid i)\right\} \\
7 & \mathrm{O}^{\mathrm{p}} \neg i & 1 ;(\overline{\mathrm{FDp}})^{c} & \left\{\mathrm{O}(\neg i \mid \mathrm{T}) \wedge{ }_{\mathrm{P}} \mathrm{O}(\neg i \mid \mathrm{T})\right\} \\
8 & \mathrm{O}^{\mathrm{p}} m & 3,4 ;(\overline{\mathrm{FDp}})^{c} & \left\{\mathrm{O}(m \mid i) \wedge{ }^{\mathrm{p}} \mathrm{O}(m \mid i)\right\}
\end{array}
$$

Note that $(\overline{\mathrm{Ep}})$ is not applicable to $\mathrm{O}(m \mid i)$ and $i$ in order to derive $\stackrel{\mathrm{p}}{\neg} \mathrm{O}(\neg i \mid \top)$ since we are missing the additional, though counter-intuitive premise $\mathrm{P}(i \mid \mathrm{T})$. In case of the first proof we have ${ }^{\mathrm{P}} \mathrm{O}(m \mid i)$, since (CTDR) is applied to $\mathrm{O}(\neg i \wedge \neg m \mid \mathrm{T})$ and $\mathrm{O}(m \mid i)$. In the second proof however we have no means to derive $\mathrm{O}(\neg i \wedge \neg m \mid$ $\top)$. Therefore the conditional application of $(\overline{F D p})$ to $\mathrm{O}(m \mid i)$ is not blocked and thus $\mathrm{O}^{\mathrm{P}} m$ is finally derivable. Not so in the first proof, where this application is blocked at line 10. However here $\mathrm{O}(\neg m \mid \mathrm{T})$ is derivable and $(\overline{\mathrm{FDp}})$ is applied to this obligation: as a consequence we arrive at the proper obligation $\mathrm{O}^{\mathrm{p}} \neg m$ at line 14.

What is the correct result? What about the solution offered by DCDPMd? This logic allows for the following versions of deontic detachment (DD):23

$$
\begin{aligned}
& \frac{\mathrm{O}(A \mid C) \quad \mathrm{P}(A \wedge B \mid C) \quad \mathrm{O}(B \mid A \wedge C)}{\mathrm{O}(B \mid C)} \\
& \frac{\mathrm{O}(A \mid \top) \mathrm{P}(A \wedge B \mid \top) \mathrm{O}(B \mid A)}{\mathrm{O}(B \mid \mathrm{\top})}
\end{aligned}
$$

This enables the logic to derive $\mathrm{O}(\neg m \mid \top)$ from the given obligations $\mathrm{O}(\neg i \mid \top)$ and $\mathrm{O}(\neg m \mid \neg i)$ (and the harmless permission statement $\mathrm{P}(\neg i \wedge \neg m \mid \top)$ ). From this we immediately get $\mathrm{O}(\neg i \wedge \neg m \mid \mathrm{T})$ which makes $\mathrm{O}(m \mid i)$ a strong CTD-obligation. Note that the commitment $\mathrm{O}(\neg m \mid \neg i)$ not to marry Diane, which is given as premise on the condition that he doesn't impregnate her, is in this logic treated as the general obligation not to marry her, $\mathrm{O}(\neg m \mid \mathrm{T})$, which would be violated in case he impregnates her. Indeed, $i$ is a violation context for $\mathrm{O}(\neg m \mid \mathrm{T})$ since we have $\mathrm{O}(m \mid i)$ and $\mathrm{O}(\neg i \mid \mathrm{T})$ which is equivalent to $\neg \mathrm{P}(i \mid \mathrm{T}){ }^{24}$ Thus, in this logic the commitment not to marry her is a proper obligation which is in conflict with the instrumental CTD-obligation to marry her. The latter is in force since its condition, him having impregnated her, is fulfilled. More generally speaking: the logic elevates, via deontic detachment, commitments on conditions stating that a proper obligation is fulfilled, such as $\mathrm{O}(\neg m \mid \neg i)$, to be proper obligations, e.g. $\mathrm{O}^{\mathrm{P}} \neg m$. Deontic detachment has quite some intuitive appeal as well as the thought that, if $A$ is a proper obligation and you are committed to bring about $B$ in case $A$, then also $B$ should be a candidate for being a proper obligation.

However, this is not so in the second logic. Indeed, there is an alternative intuition. In the case of DCDPMe the deontic detachment is blocked, since this logic only supports a weaker form of (DD):

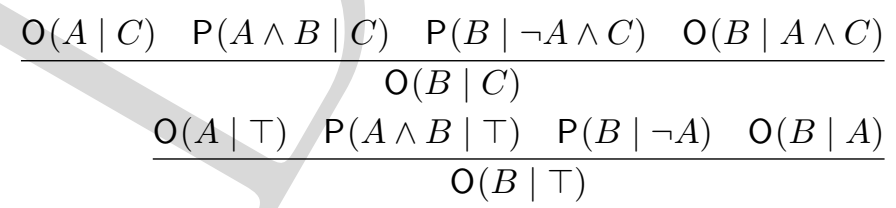

\footnotetext{
${ }^{23}$ The second is a consequence of the first one, which can easily be shown.

${ }^{24}$ This refers to case (b) in our definition of violation contexts. It is interesting to notice that violation contexts of type (b) give rise to CTD-obligations (in case the aggregation principle is valid): in our example $i$ defines a violation context to $\mathrm{O}(\neg m \mid \top)$ since we have $\mathrm{O}(m \mid i)$ and $\mathrm{O}(\neg i \mid \mathrm{\top})$. By aggregation we get $\mathrm{O}(\neg m \wedge \neg i \mid \mathrm{T}) . \mathrm{O}(m \mid i)$ is a strong CTD-obligation to the latter.
} 
In favour of this approach may be argued that, after all, the obligation not to marry her was stated only on the condition that he doesn't impregnate her. This is clearly weaker than $\mathrm{O}(\neg m \mid T)$, not to marry her in general. While it seems harmless to derive this commitment in case of $\mathrm{P}(\neg m \mid i)$, in our case it is critical. After all $\mathrm{O}(m \mid i)$ is only a weak CTD-obligation to $\mathrm{O}(\neg i \mid \mathrm{T})$ : thus to marry her in case of him impregnating her shouldn't be considered as a violation of a primary obligation (unlike the gentle killing in the Forrester paradox) but rather as a proper obligation in a violation context, similarly to our intuition which tells us in the (PA) example that it is not just an instrumental but rather a proper obligation to apologize in sub-ideal situation of the broken promise. This logic reflects therefore the difference between stating $\mathrm{O}(\neg m \mid \mathrm{T})$ and stating $\mathrm{O}(\neg m \mid \neg i)$ on the level of proper and instrumental obligations.

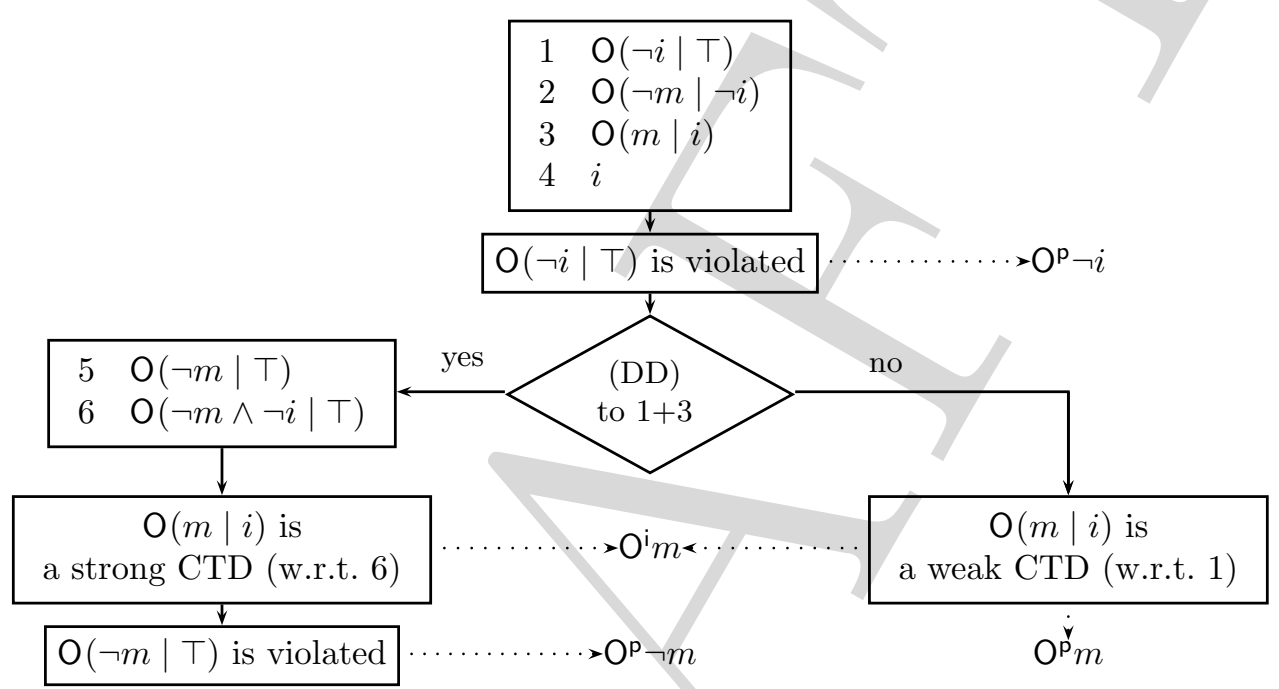

Figure 2: Two ways of dealing with Chisholm's problem. Left: the treatment in DCDPMd, Right: the treatment in DCDPMe.

I leave it to the reader to settle for one of the two intuitions and pick the corresponding logic.

I add one more example (F) to underline the role played by deontic detachment and also strengthening of the antecedent in the derivation of proper and instrumental obligations.

F1 There must be no fence. $-\mathrm{O}(\neg f \mid T)$

F2 There must be a white fence, if there is a fence. $-\mathrm{O}(w \wedge f \mid f)$

F3 There must be a fence, if there is a dog.- $\mathrm{O}(f \mid d)$

F4 It is allowed to have a dog.- $\mathrm{P}(d \mid \mathrm{T})$

F5 There is a fence and a dog. $-f \wedge d$

Let us take a look at a proof for example $(\mathrm{F})$ :

$\begin{array}{llll}1 & \mathrm{O}(\neg f \mid \top) & \text { PREM } & \emptyset \\ 2 & \mathrm{O}(w \wedge f \mid f) & \text { PREM } & \emptyset \\ 3 & \mathrm{O}(f \mid d) & \text { PREM } & \emptyset\end{array}$




\begin{tabular}{|c|c|c|}
\hline $\mathrm{P}(d \mid \top)$ & PREM & $\emptyset$ \\
\hline$d$ & PREM & $\emptyset$ \\
\hline${ }^{\mathrm{p}} \mathrm{O}(w \wedge f \mid f)$ & 1,$2 ;(\mathrm{CTDR})$ & $\emptyset$ \\
\hline${ }^{\mathrm{p}} \mathrm{O}(\neg f \mid \mathrm{\top})$ & $1,3,4,5 ;(\mathrm{Ep})$ & $\emptyset$ \\
\hline$\stackrel{\mathrm{i}}{\mathrm{O}}(\neg f \mid \mathrm{T})$ & $1,3,5 ;(\mathrm{oV}-\mathrm{Ei})$ & $\emptyset$ \\
\hline$O^{p} f$ & $3,5,(\mathrm{FDp})^{c}$ & $\{\mathrm{O}(f \mid d) \wedge \stackrel{\mathrm{p}}{\neg} \mathrm{O}(f \mid d)\}$ \\
\hline $\mathrm{O}^{\mathrm{i} f}$ & $3,5:(\overline{\mathrm{FDi}})^{c}$ & $\{\mathrm{O}(f \mid d) \wedge \stackrel{\mathrm{i}}{ } \mathrm{O}(f \mid d)\}$ \\
\hline
\end{tabular}

So far the logic derives the desired consequences. However, the reader might wonder if not also the obligation to bring about $w \wedge f$ should be detached. We cannot apply detachment to $\mathrm{O}(w \wedge f \mid f)$ since its condition is not fulfilled and even if that were so, we derived ${ }^{\mathrm{p}} \mathrm{O}(w \wedge f \mid f)$. Let us add the harmless premise $\mathrm{P}(w \wedge f \mid d): 25$

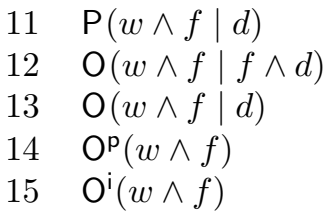

PREM 2,11; (WRM) 3,11,12;(DDP1) 5,$13 ;(\mathrm{FDp})^{c}$ 5,$13 ;(\overline{\mathrm{FDi}})^{c}$

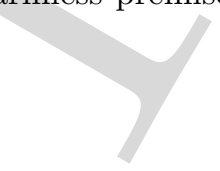

$\emptyset$

$\{\mathrm{O}(w \wedge f \mid d) \wedge \stackrel{\mathrm{p}}{\neg} \mathrm{O}(w \wedge f \mid d)\}$ $\left\{\mathrm{O}(w \wedge f \mid d) \wedge{ }^{\mathrm{i}} \mathrm{O}(w \wedge f \mid d)\right\}$

The reader can see that by strengthening of the antecedent at line 12 the obligation in question can be derived on the more restricted condition $f \wedge d$. This enables us to apply deontic detachment in order to arrive at $\mathrm{O}(w \wedge f \mid d)$. Now the detachment cannot be blocked anymore. Thus, despite the fact that $\mathrm{O}(w \wedge f \mid f)$ is a CTD-obligation, this obligation can be detached since it can be derived also for the context $d$, which is the factual situation presented by the premises. This behaviour of the logic is intuitive.

It is left to the reader to proof that in case F5 is replaced by $f \wedge \neg d$ we get $\mathrm{O}^{\mathrm{p}} \neg f$ and $\mathrm{O}^{\mathrm{i}}(w \wedge f)$ as consequences. This is as expected, since "[w] hat is most striking about the fence example [...] is the observation that when the premise $\mathrm{O}(\neg f \mid \mathrm{T})$ is violated by $f$, then the obligation for $\neg f$ should be derivable, but not when $\mathrm{O}(\neg f \mid \top)$ is overridden by the exception $f \wedge d$ " ([20] p. 71).

\section{Some enhancements and refinements}

In this section I introduce some useful ways of enhancing our adaptive system. Due to space limitations the explications in this section are more of a suggestive nature though they are precise and explicit enough to guide the interested reader in a concrete realization.

\subsection{More adaptiveness}

Applying aggregation and inheritance conditionally As pointed out in [17] and [16], one of the disadvantages of Goble's monadic logics DPM and of his dyadic generalizations CDPM is that, in order to apply inheritance and aggregation, additional premises have to be added by the user of the logic. This is sub-optimal in the sense that as much reasoning as possible should be performed by the logic and, inversely, as less as possible should be left to the user. Especially in complicated settings the adding of premises can be very bothersome for the user, since in such cases it is in no way a trivial question whether the addition of a certain permission statement leads to explosion or otherwise unintuitive consequences.

In [17] and [16] adaptive logics on the basis of Goble's systems were developed which perform this task instead of the user: inheritance and aggregation are applied conditionally. Remember, that in order to achieve tolerance concerning deontic

\footnotetext{
${ }^{25}$ In DCDPMe we would have to add another additional premise, $\mathrm{P}(w \wedge f \mid d \wedge \neg f)$, in order to derive $\mathrm{O}(w \wedge f \mid d)$ analogously.
} 
conflicts, Goble added the constraint $\mathrm{P}(A \wedge B \mid C)$ to the aggregation rule of standard deontic logic. This means that the aggregation is applied only in cases where it does not produce a deontic conflict. The idea of the adaptive logic is now to apply aggregation on the condition that no deontic conflict would result from it. Therefore the set of abnormalities $\Omega^{\wedge}={ }_{\mathrm{df}}\{\mathrm{O}(A \mid C) \wedge \mathrm{O}(B \mid C) \wedge \mathrm{O}(\neg(A \wedge B) \mid$ $C) \mid A, B, C \in \mathcal{P}\}$ and the minimal abnormality strategy are employed. Note that the following is valid in Goble's CDPM systems:

$\mathrm{O}(A \mid C) \wedge \mathrm{O}(B \mid C) \vdash \mathrm{O}(A \wedge B \mid C) \vee(\mathrm{O}(A \mid C) \wedge \mathrm{O}(B \mid C) \wedge \mathrm{O}(\neg(A \wedge B) \mid C))$

This allows us to derive $\mathrm{O}(A \wedge B \mid C)$ from $\mathrm{O}(A \mid C)$ and $\mathrm{O}(B \mid C)$ on the condition $\{\mathrm{O}(A \mid C) \wedge \mathrm{O}(B \mid C) \wedge \mathrm{O}(\neg(A \wedge B) \mid C)\}$. Similarly, as the reader may easily verify, this enables us to apply inheritance conditionally: in case $A \vdash B$, from $\mathrm{O}(A \mid C)$ we get $\mathrm{O}(B \mid C)$ on the condition $\{\mathrm{O}(A \mid C) \wedge \mathrm{O}(A \mid C) \wedge \mathrm{O}(\neg A \mid C)\}$.

By adjusting the marking conditions, it is technically very straightforward to realize a sequential adaptive logic, with this logic being the first and our adaptive logic for detachment being the second logic. This way the advantages of both systems may be combined.

Let us take another look at the proof for the Chisholm example on page 14 . In order to derive $\mathrm{O}(\neg i \wedge \neg m \mid \top)$ from $\mathrm{O}(\neg i \mid T)$ and $\mathrm{O}(\neg i \supset \neg m \mid \mathrm{T})$ by (CPAND) we had to introduce the additional permission statement $\mathrm{P}(\neg i \wedge \neg m \mid \top)$ at line 7. Furthermore, in order to apply inheritance to $\mathrm{O}(\neg i \wedge \neg m \mid \mathrm{T})$ to derive $\mathrm{O}(\neg m \mid \top)$ we were again in need of $\mathrm{P}(\neg i \wedge \neg m \mid \top)$. However, using the adaptive logic introduced in this section, the user is not in need of adding this permission by hand. The logic conditionally applies aggregation and inheritance as follows (where $X=\mathrm{O}(\neg i \mid \top) \wedge \mathrm{O}(\neg i \supset \neg m \mid \top) \wedge \mathrm{O}(\neg(\neg i \wedge \neg m) \mid \top))$ :

$$
\begin{array}{llll}
7^{\prime} & \mathrm{O}(\neg i \wedge \neg m \mid \top) & 1,6 ;\left(\mathrm{CPAND}^{c}\right. & \{X\} \\
8^{\prime} & \mathrm{O}(\neg m \mid \top) & 7 ;(\overline{\mathrm{RCPM}})^{c} & \{X\}
\end{array}
$$

Applying (SA) conditionally Lou Goble's version of strengthening the antecedent is not without problems. Take for instance the asparagus example, $\mathrm{O}(\neg f \mid$ $\top)$ and $\mathrm{P}(f \mid a)$, and add the, in no way counter-intuitive, premise $\mathrm{P}(\neg f \wedge a \mid \top)$. This leads to explosion applying (WRM) to $\mathrm{O}(\neg f \mid \top)$ and $\mathrm{P}(\neg f \wedge a \mid \top)$, as the reader can easily verify. This demonstrates that Goble's system is not fully able to deal with specifity cases.

Analogously to the adaptive treatment of the inheritance principle we can emply an adaptive logic to apply (SA) as much as possible. We therefore define the set of abnormalities $\Omega^{\mathrm{SA}}=\mathrm{df}_{\mathrm{df}}\{\mathrm{O}(B \mid A) \wedge \mathrm{P}(B \mid A \wedge C) \wedge \neg \mathrm{O}(B \mid A \wedge C)\}$. The strategy is minimal abnormality and as lower limit one of the CDPM variants introduced in this paper can be used. In this logic it is possible to conditionally derive $\mathrm{O}(B \mid A \wedge C)$ from $\mathrm{O}(B \mid A)$ and $\mathrm{P}(B \mid A \wedge C)$.

Again, this system can, in a straightforward way be combined with the other adaptive logics introduced this paper. This is left as an easy exercise to the reader.

\subsection{Deontic conflicts}

Our logic DCDPM has a feature that some readers might find troublesome: conflicts at the level of conditional obligations may be inherited to the level of proper or instrumental obligations. Take for instance the conflicting setting $\mathrm{O}(a \mid T)$ and $\mathrm{O}(b \mid \mathrm{T})$ (where $a \vdash \neg b)$. In this case $\mathrm{O}^{\mathrm{p}} a \wedge \mathrm{O}^{\mathrm{p}} b$ is a DCDPM-consequence (and analogously $\mathrm{O}^{\mathrm{i}} a \wedge \mathrm{O}^{\mathrm{i}} b$ ). There are reasons to see this as counter-intuitive or dubious on philosophical grounds: we should not have conflicting proper or instrumental obligations. This defect is not fatal, the logic can be adjusted in a way to avoid such 
consequences. Therefore we alter the rules for factual detachment in the following way (where $x \in\{p, i\}$ ):

$$
\frac{\mathrm{O}(A \mid B) \quad \mathrm{P}(A \mid B) \quad B \quad \neg \stackrel{\times}{ } \mathrm{O}(A \mid B)}{\mathrm{O}^{\times} A}
$$

Rule $(\overline{\mathrm{FDPx}})$ ensures by means of the additional constraint $\mathrm{P}(A \mid B)$ that factual detachment is only applied to non-conflicting conditional obligations.

Not do derive any actual obligation in the face of two conflicting obligations which are not overridden is of course too weak. But note that in our adaptive strengthenings of Goble's CDPM introduced in Section 6.1, we can derive for instance $\mathrm{O}(a \vee b \mid T) \wedge \mathrm{P}(a \vee b \mid T)$ from $\mathrm{O}(a \mid T)$ and $\mathrm{O}(b \mid \mathrm{T})$. This enables us to derive $\mathrm{O}^{\times}(a \vee b)$ by (FDPx). This certainly is as desired.

Similarly in the case that $\mathrm{O}(a \mid \mathrm{T})$ is being overridden by $\mathrm{O}(\neg a \mid c)$ and $c$ : by our adaptive handling of (SA) introduced in Section 6.1 we are able to derive $\mathrm{O}(b \mid c)$ in case we know that $b$ is allowed under the circumstances described by $c$. In this case we apply (FDPx) to $\mathrm{O}(b \mid c)$ and $\mathrm{P}(b \mid c)$ and derive the desired $\mathrm{O}^{\times} b$.

\section{Conclusion}

In this paper I proposed a generic way to turn a given dyadic deontic logic into an adaptive logic which models detachment. I elaborated two intuitions concerning what obligations should be detached: proper and instrumental obligations. Obligations which are not overridden by more specific obligations bind us also in cases in which they are violated and are therefore considered to be proper obligations. Instrumental obligations are a more pragmatic concept: taking the factual premises as immutable, we can ask ourselves the question, what is the best thing to do? For instance contrary-to-duty obligations indicate instrumental obligations, while the primary obligations which may be violated do not cease to bind as as proper obligations. Paradigmatically I presented an adaptive logic based on Lou Goble's conflict-tolerant CDPM system which implements the ideas presented before.

\section{APPENDIX}

Semantics for the dyadic systems CDPM.1c and CDPM.2c are introduced by Goble in [5]. As in [17] we use an actual world variant of the semantics in order to deal with factual premises.

Let a dyadic neighborhood frame $F$ be a pair $\left\langle W, w_{a}, \mathcal{O}\right\rangle$ where $W$ is a set of worlds, $w_{a} \in W$ is the actual world, and $\mathcal{O}$ assigns to each world $a \in W$ a set of ordered propositions, i.e. $\mathcal{O}_{a} \subseteq \wp W \times \wp W$. A model $M$ on a frame $F$ is a pair $\langle F, v\rangle$ where $v(p) \subseteq W$ for each propositional letter $p$. We define $M \models \varphi$ iff $M, w_{a} \models \varphi$. Furthermore, where $a \in W$, we have the following requirements:

$$
\begin{array}{r}
M, a \models p \text { iff } a \in v(p), \text { where } p \text { is a propositional letter } \\
M, a \models \mathrm{O}(A \mid B) \text { iff }\left\langle|B|_{M},|A|_{M}\right\rangle \in \mathcal{O}_{a} \\
\left\langle|B|_{M},|A|_{M}\right\rangle \in \mathcal{O}_{a} \text { or } M, a \models{ }^{\times} \mathrm{O}(A \mid B) \text { where } \mathrm{x} \in\{\mathrm{i}, \mathrm{p}\} \\
M, a \models \mathrm{P}(A \mid B) \text { iff }\left\langle|B|_{M}, \overline{|A|_{M}}\right\rangle \notin \mathcal{O}_{a}
\end{array}
$$

where $|A|_{M}={ }_{\mathrm{df}}\{a \in W \mid M, a \models A\}$ and $\overline{W^{\prime}}={ }_{\mathrm{df}} W \backslash W^{\prime}$ for $W^{\prime} \subseteq W$. For the classical connectives the definitions are as usual:

$$
\begin{array}{r}
M, a \models \neg \varphi \text { iff } M, a \not \models \varphi \\
M, a \models \varphi \vee \psi \text { iff } M, a \models \varphi \text { or } M, a \models \psi \\
M, a \models \varphi \wedge \psi \text { iff } M, a \models \varphi \text { and } M, a \models \psi \\
M, a \models \varphi \supset \psi \text { iff } M, a \models \neg \varphi \vee \psi
\end{array}
$$


In order to define our CDPM systems we also need the following rules:

$$
\begin{aligned}
& \langle W, W\rangle \in \mathcal{O}_{a} \\
& \text { If } Y \subseteq Z \text { and }\langle X, Y\rangle \in \mathcal{O}_{a} \text { and }\langle X, \bar{Y}\rangle \notin \mathcal{O}_{a} \text { then }\langle X, Z\rangle \in \mathcal{O}_{a} \\
& \text { If }\langle X, Y\rangle \in \mathcal{O}_{a} \text {, for any } Y \subseteq W \text {, then }\langle X, X\rangle \in \mathcal{O}_{a} \\
& \text { If }\langle X \cap Y, Z\rangle \in \mathcal{O}_{a} \text {, then }\langle X, \bar{Y} \cup Z\rangle \in \mathcal{O}_{a} \\
& \text { If }\langle X, Y\rangle \in \mathcal{O}_{a} \text { and }\langle X, \overline{Y \cap Z}\rangle \notin \mathcal{O}_{a} \text {, then }\langle X \cap Z, Y\rangle \in \mathcal{O}_{a} \\
& \text { If }\langle X, Y\rangle \in \mathcal{O}_{a},\langle X, Z\rangle \in \mathcal{O}_{a} \text { and }\langle X, \overline{Y \cap Z}\rangle \notin \mathcal{O}_{a} \text {, then }\langle X, Y \cap Z\rangle \in \mathcal{O}_{a} \\
& \langle X, \emptyset\rangle \in \mathcal{O}_{a}
\end{aligned}
$$

For the $f$-version of our system we add

$$
\text { If }\langle X \cap Z, Y\rangle \in \mathcal{O}_{a} \text { and }\langle\bar{Y} \cap Z, \bar{X}\rangle \notin \mathcal{O}_{a} \text {, then }\langle Z, \bar{Y} \cup X\rangle \in \mathcal{O}_{a}
$$

The following rules give an accurate account of detachment:

$$
\begin{aligned}
& \text { If } M, a \models B, \mathrm{O}(A \mid B) \text { and } M, a \not \models \stackrel{\mathrm{x}}{\neg} \mathrm{O}(A \mid B) \text { then } M, a \models \mathrm{O}^{\times} A \\
& \text { If } M, a \models B \wedge C, \mathrm{P}(C \mid B), \mathrm{O}(A \mid B), \mathrm{P}(D \mid B \wedge C) \vee \mathrm{O}(D \mid B \wedge C) \\
& \text { and }|D|_{M} \subseteq \overline{|A|_{M}} \text {, then } M, a \models \stackrel{\mathrm{p}}{\neg} \mathrm{O}(A \mid B) \\
& \text { If } M, a \models \mathrm{O}(A \mid B \wedge C), \mathrm{O}(D \mid B) \text {, and }|D|_{M} \subseteq \overline{|B \wedge C|_{M}},|D|_{M} \subseteq \overline{|A|_{M}} \text {, } \\
& \text { then } M, a \models{ }^{\mathrm{p}} \mathrm{O}(A \mid B \wedge C)
\end{aligned}
$$

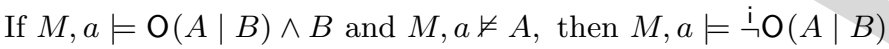

$$
\begin{aligned}
& \text { If } M, a \models B \wedge C, \mathrm{O}(A \mid B), \mathrm{P}(D \mid B \wedge C) \vee \mathrm{O}(D \mid B \wedge C) \\
& \text { and }|D|_{M} \subseteq \overline{|A|_{M}} \text {, then } M, a \models \text { i } \mathrm{O}(A \mid B)
\end{aligned}
$$

Theorem 1. Logics CDPM.2c, CDPM.2d and CDPM.2e are sound and complete with respect to their corresponding class of frames which are as follows: For CDPM.2c we take rules $(\overline{\mathrm{S}-\mathrm{p})}),(\mathrm{S}-\neg),(\mathrm{S}-\mathrm{V}),(\mathrm{S}-\wedge),(\mathrm{S}-\mathrm{D}),(\mathrm{S}-\mathrm{O}),(\mathrm{S}-\mathrm{P}),(\mathrm{S}-\mathrm{CN}),(\mathrm{S}-\mathrm{CPAND})$, $(\mathrm{S}-\mathrm{RCPM})$, (S-QR), (S-S), (S-WRM). For CDPM.2d we disregard (S-QR) and add furthermore $\overline{(\mathrm{S}-\mathrm{Ox})},(\mathrm{S}-\mathrm{FDx})$ (for $\mathrm{x} \in\{\mathrm{i}, \mathrm{p}\})$, (S-Ep), (S-CTDR), (S-fV) and (S-oV-Ei). CDPM.2e is like CDPM.2d with the exception of (S-S) which is replaced by (S-PS').

Proof. This can be proven analogous to the way completeness and correctness was proven in [5] for CDPM.2c.

\section{References}

[1] Diderik Batens. A universal logic approach to adaptive logics. Logica Universalis, 1:221-242, 2007.

[2] Roderick M. Chisholm. Contrary-to-duty imperatives and deontic logic. Analysis, 24:33-36, 1963.

[3] Philippa Foot. Moral realism and moral dilemma. The Journal of Philosophy, 80(7):379-398, 1983.

[4] J. Forrester. Gentle murder, or the adverbial samaritan. The Journal of Philosophy, 81:193$197,1984$.

[5] Lou Goble. Dilemmas in deontic logic. To appear.

[6] Lou Goble. Preference semantics for deontic logic part i - simple models. Logique $\&$ Analyse, pages 383-418, 2003.

[7] Lou Goble. A logic for deontic dilemmas. Journal of Applied Logic, 3:461-483, 2005.

[8] Patricia S. Greenspan. Conditional ought and hypothetical imperatives. Journal of Philosophy, 72:259-276, 1975.

[9] Daniel Lehmann and Menachem Magidor. What does a conditional knowledge base entail? Artificial Intelligence, 55(1):1-60, 1992.

[10] David Lewis. Counterfactuals. Blackwell Publishers, December 2000. 
[11] Barry Loewer and Marvin Belzer. Dyadic deontic detachment. Synthese, 54:295-318, 1983.

[12] Paul McNamara. Deontic logic. In Dov Gabbay and John Woods, editors, Handbook of the History of Logic, pages 197-288. Elsevier, 2007.

[13] Henry Prakken and Marek Sergot. Contrary-to-duty obligations. Studia Logica, 57(1):91115, July 1996.

[14] Henry Prakken and Marek Sergot. Dyadic deontic logic and contrary-to-duty obligations. In D. Nute, editor, Defeasible Deontic Logic, pages 223-262. Kluwer, 1997.

[15] Lennart Åqvis. Deontic logic. In D. Gabbay and F. Guenthner, editors, Handbook of Philosophical Logic, pages 605-714. Reidel, Dordrecht, 1984.

[16] Christian Straßer. An adaptive logic framework for conditional obligations and deontic dilemmas. Forthcoming. Presented at the WCP4, Melbourne, 2008.

[17] Christian Straßer and Joke Meheus. Adaptive versions of the deontic logics DPM. To appear. Presented at CLMPS 2007, Beijing.

[18] Yao-Hua Tan. Cancelling and overshadowing: two types of defeasibility in defeasible deontic logic. In In Proceedings of the Fourteenth International Joint Conference on Artificial Intelligence (IJCAIË\#039;95, pages 1525-1532, 1995.

[19] Leendert van der Torre. Violation contexts and deontic independence. In In Modeling and Using Context. Proceedings of the CONTEXTE\#039;99, Lecture Notes in Artificial Intelligence 1688, pages 361-374, 1999.

[20] Leendert van der Torre and Yao H. Tan. Contrary-to-duty reasoning with preference-based dyadic obligations. Annals of Mathematics and Artificial Intelligence, 27(1-4):49-78, 1999.

[21] Bas van Fraassen. The logic of conditional obligation. Journal of Philosophical Logic, 1:417$438,1972$.

[22] Linton Wang. The detachment problem of conditional obligations. Forthcoming.

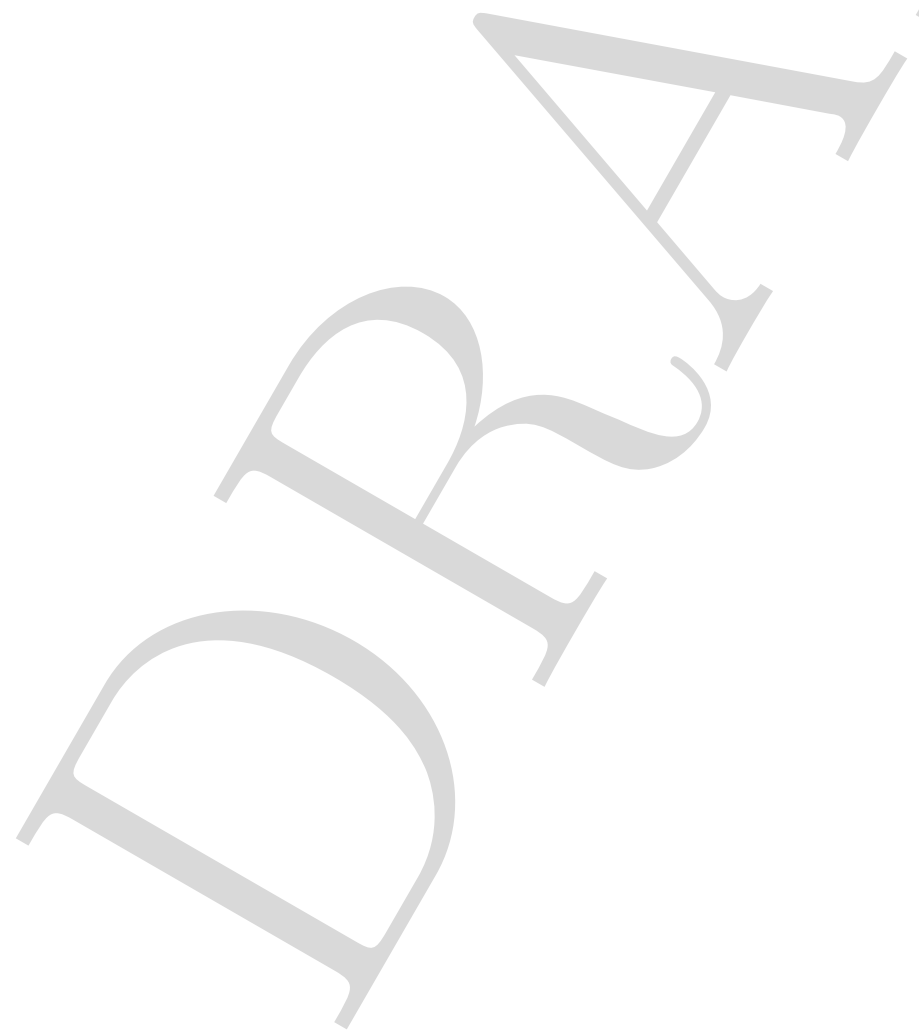

